\title{
Biological Evolution: Entropy, Complexity and Stability
}

\author{
Charu Gopal Chakrabarti, Koyel Ghosh \\ Department of Applied Mathematics, University of Calcutta, Kolkata, India \\ E-mail: cgc_math@rediffmail.com,koyelghosh1983@gmail.com \\ Received December 28, 2010; revised March 28, 2011; accepted April 10, 2011
}

\begin{abstract}
In the present paper we have made an attempt to investigate the importance of the concepts of dynamical stability and complexity along with their interrelationship in an evolving biological systems described by a system of kinetic (both deterministic and chaotic) equations. The key to the investigation lies in the expression of a time-dependent Boltzmann-like entropy function derived from the dynamical model of the system. A significant result is the determination of the expression of Boltzmann-entropy production rate of the evolving system leading to the well-known Pesin-type identity which provides an elegant and simple measure of dynamical complexity in terms of positive Lyapunov exponents. The expression of dynamical complexity has been found to be very suitable in the study of the increase of dynamical complexity with the successive instabilities resulting from the appearance of new polymer species (or ecological species) into the original system. The increase of the dynamical complexity with the evolutionary process has been explained with a simple competitive model system leading to the "principle of natural selection".
\end{abstract}

Keywords: Boltzmann-Like Entropy, Dynamical Stability, Pesin-Type Identity and Dynamical Complexity, Lyapunov Exponents, Structural Instabilities, Biological Evolution

\section{Introduction}

The evolution in physical science is referred to the approach of a system to the thermodynamic equilibrium characterized by the increase of entropy of the second law of thermodynamics. The evolution in physical system is always directed to the continuous disorganization, that is, to the destruction of structures introduced by the initial conditions [1-3]. The biological evolution, on the other hand, points precisely to the opposite direction. In biology the idea of evolution is associated with the irreversible increase of organization giving rise to the creation of more and more complex structures. These two aspects of evolution can be reconciled by the concept of the open-system model of living system and the application of the second law of thermodynamics to the system as a whole-living + environment [1-3]. Like in physical science the entropy plays a significant role in biological evolution [4-12]. Both organization and complexity can be measured in terms of entropy. Another important idea which play significant role in the study of evolution is the concept of stability. The evolution can, in fact, be viewed as a problem of stability [1] and it can also be considered as the process that generates most of all, if not all, complex structures in nature [8]. Both the con- cepts of stability and complexity are interrelated playing significant role in the process of evolution as we are going to investigate.

In the present paper we have made an attempt to study the importance of the concepts of stability and complexity together with their relationship in the characterization of evolution of a biological system. We have considered first a dynamical model of the biological system consisting of a number of interacting polymer species (or ecological species) described by a system of non-linear rate equations. We have studied the local dynamical behaviours of the system such as the criteria of stability and complexity around a stationary state. We have next considered a statistical mechanical model of the system around the stationary state and found out the expression of Boltzmann-like entropy of the evolving system. The rate of change of Boltzmann-entropy, that is, Boltzmann-entropy production rate leads to the well-known Pesin-type identity which provides an elegant and simple measure of dynamical complexity in terms of positive Lyapunov exponents [17-19]. The expression of dynamical complexity has been found to be very suitable in the study of the increase of complexity with the successive instabilities resulting from the appearance of new polymer species (or ecological species) into the original 
system. The increase of the dynamical complexity with the evolutionary process has been explained with a simple competitive model system leading to the "principle of natural selection".

\section{Biological System: Dynamical Model and Stability}

Let us consider a biological system consisting of $\mathrm{n}$ interacting components (e.g. polymer species or ecological species) of concentration $x^{i},(i=1,2, \cdots, n)$. The dynamical model consists of the set of rate equations in state space

$$
\dot{x}=f(x, \alpha)
$$

where $x=\left(x_{1}, x_{2}, \cdots, x_{n}\right)$ is a point in the n-dimensional state-space, $\alpha$ is a control parameter. For simplicity we consider a single parameter. The functions $f=\left(f_{1}\right.$, $\left.f_{2}, \cdots, f_{n}\right)$ are assumed to be continuously differentiable in some open set $S=\left\{x_{i} ; x_{i} \geq 0, i=1,2, \cdots, n\right\}$ in the state-space. The system of equations (2.1) are in general non-linear and it is difficult to find out the solution in closed form. We assume that for a certain value (or a range of values) of the control parameter $\alpha$ the system (2.1) has a stationary solution or fixed point $x^{*}=\left(x_{1} *\right.$, $\left.x_{2} *, \cdots x_{n} *\right)$ (say). We consider a neighbouring point $x(t)=x *+\delta x$, where $\delta x(t)$ is the deviation of $x(t)$ from the stationary state or fixed point $x^{*}$. Linearizing the system of Equations (2.1) about the stationary point or fixed point $x *$ the time-evolution of $\delta x(t)$ is given by

$$
\delta \dot{x}(t)=A(x *) \delta x(t)
$$

where $A(x *)=\left[\left(\partial f_{i} / \partial f_{j}\right)_{x^{*}}\right]$ is the Jacobian matrix of the function $\mathrm{f}$ at the stationary state $x *$. The time derivative in the left-hand side represents the local derivative $\delta / \delta t$ instead of the total time-derivative $\mathrm{d} / \mathrm{d} t$. The solution of (2.2) is given by

$$
\delta x(t)=e^{A t} \delta x(0)
$$

$\delta x(0)$ is the deviation of the initial state (or point) from the stationary point $x *$. For orthonormal representation of the Jacobian matrix $A(x *)$ we have

$$
A(x *)=\operatorname{Diag}\left(\lambda_{1}, \lambda_{2}, \cdots \lambda_{n}\right)
$$

where $\left(\lambda_{1}, \lambda_{2}, \cdots \lambda_{n}\right)$ are the eigenvalues of the matrix $A(x *)$. In this case the solution (2.3) reduces to the form

$$
\delta x_{i}(t)=\delta x_{i}(0) e^{\lambda_{i} t},(i=1,2, \cdots, n)
$$

The solution (2.5) shows that the asymptotic stability of the stationary state $x *$ requires that the real parts of all eigenvalues must be negative: $\operatorname{Re}\left(\lambda_{j}<0\right.$ for all $\left.\lambda_{j}\right)$. This implies that all the deviations $\delta x_{i}(t)$ regress with time for asymptotic stability. If any one of the eigenvalues has a positive real part, the stationary state is unstable. We can extend the above result for chaotic systems. For the dynamical model system (2.1) let us consider two neighbouring trajectories-one arising from the reference point $x_{r}$ and another from the neighbouring point $x(t)=x_{r}+\delta x(t)$. The distance between the two trajectories at time $t$ is given by the Euclidean norm

$$
\delta x(t)=\left[\delta x_{1}^{2}(t)+\delta x_{2}^{2}(t)+\cdots+\delta x_{n}^{2}(t)\right]^{\frac{1}{2}}
$$

For orthonormal representation of the Jacobian matrix $A(x *)$ we have

$$
\delta x_{i}(t)=\delta x_{i}(0) e^{\lambda_{i} t},(i=1,2, \cdots, n)
$$

where $\lambda_{i}(i=1,2, \cdots, n)$ is the Lyapunov exponent defined as the average rate of divergence of two neighbouring trajectories [20]:

$$
\lambda_{i}=\lim _{t \rightarrow \infty} \frac{1}{t} \log \left[\frac{\delta x_{i}(t)}{\delta x_{i}(0)}\right],(i=1,2, \cdots, n)
$$

It is important to note that the Lyapunov exponent $\lambda_{i}$, is the value of the real part of the ith eigenvalue averaged over the trajectory under study [20]. For different values (zero, negative and positive) of the Lyapunov exponents we will get different types of attractors, for examples, fixed points, limit cycles, quasiperiodic torus and chaotic in three dimensional state space [17].

\section{Statistical Model: Entropy and Dynamical Complexity}

We now consider the concept of complexity. A system consisting of a large number of interacting or interrelated elements or components is called a complex system. How to measure the complexity? There are different approaches to the concept of complexity. The entropy which is at the heart of statistical mechanics and information theory plays a vital role in the characterization of complexity [11]. For the entropic characterization of complexity we need a statistical mechanical model out of the dynamical model of the system. Statistical model is necessary in view of the enormous number of accessible microstates (or representative points) along the different trajectories from the initial state to the current state. The statistical mechanics is also necessary for the chaotic systems which are characterized by exponential separation of nearby trajectories $[18,19]$. Both the cases represent complexity about the dynamical behaviours of the system. To find out a measure of complexity we require an appropriate measure of entropy characterizing the evolution of the system from the initial state $x(0)$ to the current state $x(t)$. For the system under consideration we 
have from $(2.5)$

$$
\delta x(t)=e^{A t} \delta x(0)=B(t) \delta x(0)
$$

where $B(t)=e^{A t}$ is the matrix of evolution and plays a crucial role in evolution of the system. In the orthonormal representation the evolution matrix $e^{A t}$ can be represented as the diagonal matrix $e^{A t}=\operatorname{diag}\left(e^{\lambda_{1} t}, e^{\lambda_{2} t}, \cdots\right.$ $\left.e^{\lambda_{n} t}\right)$. The diagonal elements $e^{\lambda_{i} t},(i=1,2, \cdots, n)$ characterize the different trajectories connecting the initial state to the final (current) state. The accessible microstates along the trajectories $e^{\lambda_{i} t}$ are thus characterized by the quantities $e^{\lambda_{i} t}, \quad(i=1,2, \cdots, n)$. Then the measure of all accessible microstates lying on the trajectory $e^{\lambda_{i} t}$ can be taken to be proportional to the quantity $e^{\lambda_{i} t}$, $(i=1,2, \cdots, n)$. The quantity $\Omega(t)=\prod_{i=1}^{n} e^{\lambda_{i} t}$ is then proportional to the measure (or volume) of the totality of all accessible microstates lying on the different trajectories for evolution: $\delta x(0) \rightarrow \delta x(t)$. With this interpretation of the quantity $\Omega(t)$ we can define a Boltzmann like entropy of the macrostate consisting of all accessible microstates in the evolution $\delta x(0) \rightarrow \delta x(t)$ as

$$
H_{B}(t)=\ln \Omega(t)=\ln \prod_{i=1}^{n} e^{\lambda_{i} t}=\sum_{i=1}^{n} \lambda_{i} t
$$

where we have taken the multiplicative constant factor to be equal to unity. The quantity (3.2) is the measure of entropy associated with the evolution $\delta x(0) \rightarrow \delta x(t)$. We can approach the determination of the expression of the entropy (3.4) without any consideration of the statistical model. The complexity of evolving system lies with the entropy of the evolution matrix $B(t)=e^{A t}$. The entropy of the non-probabilistic square matrix $B(t)$, consistent with Boltzmann entropy, is given by [14]

$$
H_{B}(t)=\log |B(t)|=\log \prod_{i=1}^{n} e^{\lambda_{i} t}
$$

where $|B(t)|$ is the determinant of the diagonal matrix $e^{A t}=\operatorname{diag}\left(e^{\lambda_{1} t}, e^{\lambda_{2} t}, \cdots e^{\lambda_{n} t}\right)$. Question may arise about the physical validity of Boltzmann-like entropy for the system under consideration. The system under consideration being in the vicinity of local stationary state with a certain value of the control parameter $\alpha$, the use of Boltzmann-like entropy is justified [19]. The exponential in (3.3) with negative Lyapunov exponents $\lambda_{i}$ must be replaced by 1 , only one microstate with negative Lyapunov exponent being occupied $\delta x_{i}=0$. So the entropy (3.2) reduces to the form

$$
H_{B}(t)=\sum_{i=1}^{n^{*}} \lambda_{i} t
$$

where the summation in the right-hand side extends over all positive Lyapunov exponents only. Evidently the entropy $H_{B}(t)$ is zero at the initial time $t=0$ and it increases with the time evolution. We now proceed to measure the complexity associated with the evolution. The complexity which we call dynamical complexity is a property of the evolution of a state and not of the state itself [13]. We, therefore, define the dynamical complexity as the rate of change of the entropy (3.4), that is, the Boltzmann-entropy production rate

$$
C=\dot{H}_{B}(t)=\sum_{i=1}^{n^{*}} \lambda_{i}
$$

where the right-hand side represents the sum of all positive Lyapunov exponents. The result (3.5) is analogues to Kolmogorov-Sinai entropy rate (or simply K-S entropy) and corresponds to the well-known Pesin's identity [17,21]. Pesin-like identity plays significant role in the characterization of complexity of different type of dynamical systems, for example, hyperbolic dynamical system [22]. The complexity measure (3.5) shows that it is completely dependent on the positive Lyapunov exponents of the system. It remains bounded for chaotic attractors which may be a point, a closed curve or an unclosed but bounded orbit, even for very complex one such as Rossler's strange attractor $[15,16]$.

\section{Structural Instabilities: Increase of Complexity and Biological Evolution}

In biology and sociology the idea of evolution is associated with the increase of complexity or organization giving rise to more and more complex structure [1]. In this section we wish to study evolution on the basis of the measure of complexity (3.5). To study evolution we have to start with appropriate rate equations or model equations describing the process. We assume that the system is maintained uniformly and that there exists at least one asymptotically stable stationary solution of the model system. This implies that all deviations or fluctuations regress in time, that is, all the eigenvalues of the characteristic equation have negative real parts. We now need to incorporate structural fluctuation resulting from the appearance of a new species or mutants in the system at stable steady state. Like in many other systems, the evolution of the system under consideration depends on the control parameter $\alpha$. As the system evolves and continuously perturbed by the outside world the parameter $\alpha$ can then change smoothly or abruptly. The change of the parameter $\alpha$ generally changes the structure of the rate equations. As a result of the change of the parameter $\alpha$ the stability of the system may be disturbed with the inclusion of a new species into the system. However, in view of the smallness of the change of the parameter $\alpha$, the structural stability of rate equations is assumed not to be disturbed, the enlarged system evolves eventually to a new stationary state, reached sooner or later depending 
on the magnitude of the changing parameter $\alpha[1,23]$. We have to study the increase of complexity (under certain condition) of the enlarged system with the successive instabilities resulting from the successive appearance of new species. According to the measure of complexity (3.5) the increase of complexity is equivalent to the appearance of a new positive Lyapunov exponent or an eigenvalue with positive real part. Let us explain this with a simple competitive model system. Let us first consider a single polymer species (or an ecological species) of concentration $x_{1}$ in a medium of limited resources. The dynamical equation of this reference species is assumed to be governed by the logistic growth equation

$$
\dot{x}_{1}=x_{1}\left\{a-b x_{1}\right\}
$$

with stationary states $x_{1}^{*}=\{a / b, 0\}$. The stationary state 0 is unstable where as $a / b$ is stable. The point $a / b$ thus represents the stable steady state of the species $x_{1}$.

Now we suppose that a new species appears by mutation or a new species invades the system. Let $x_{2}$ be the concentration of the new species at some time $t$ and is in competition with the original species $x_{1}$ for a limited resource. Let the governing equations for the whole system be given by the Lotka-Volterra model equations of competition (for simplicity excluding the case of competition exclusion) [24].

$$
\begin{aligned}
& \dot{x}_{1}=x_{1}\left\{a_{1}-b_{1}\left(x_{1}+x_{2}\right)\right\} \\
& \dot{x}_{2}=x_{2}\left\{a_{2}-b_{2}\left(x_{1}+x_{2}\right)\right\}
\end{aligned}
$$

The system (4.2) has three stationary states $\left\{s_{1}, s_{2}, s_{3}\right\}$

$$
s_{1}=(0,0), s_{2}=\left(\frac{a_{1}}{b_{1}}, 0\right), s_{3}=\left(0, \frac{a_{2}}{b_{2}}\right)
$$

The first stationary state $s_{1}=(0,0)$ is trivial and unstable. The second stationary state $s_{2}=\left(a_{1} / b_{1}, 0\right)$ consists of the population of the first species only and corresponds to the moment of the appearance of the second species or external disturbance. The system can then evolve if the state $s_{2}=\left(a_{1} / b_{1}, 0\right)$ is unstable which requires the positivity of the Lyapunov exponent or positivity of the real part of the eigenvalue. This requires

$$
\frac{b_{1} a_{2}-a_{1} b_{2}}{b_{1}}>0 \text { or } \frac{a_{2}}{b_{2}}>\frac{a_{1}}{b_{1}}
$$

This is the condition of growth of the second species $x_{2}$ and the second species $x_{2}$ grows to some finite value $a_{2} / b_{2}$. The total system then evolves to the new (third) stationary state $s_{3}=\left(0, a_{2} / b_{2}\right)$ implying the extinction of the first species $x_{1}$. The third stationary $s_{3}=\left(0, a_{2} / b_{2}\right)$ is stable if $a_{2} / b_{2}>a_{1} / b_{1}$. With this criteria of stability of the third stationary state $s_{3}$ we can now introduce a new species $x_{3}$ to the state $s_{3}=\left(0, a_{2} / b_{2}\right)$ to disturb its stability. The criteria of instability of the system with the inclusion of the third new species $x_{3}$ requires

$$
\frac{a_{3}}{b_{3}}>\frac{a_{2}}{b_{2}}
$$

The process may go on with successive instabilities of the stationary states with the appearance of new species. Note that the appearance of successive instabilities imply the appearance of successive eigenvalues with positive real parts (or positive Lyapunov exponents) and hence the increase of complexity step by step.In ecology this transitional process corresponds to the process of ecological succession [24,25]. This result may be interpreted in another way: the system tends in the long run to the stationary state characterized by the maximum of the fitness function $a_{i} / b_{i}$ :

$$
\frac{a_{1}}{b_{1}}<\frac{a_{2}}{b_{2}}<\frac{a_{3}}{b_{3}}<\cdots<\frac{a_{n}}{b_{n}}<\cdots
$$

which is nothing but the Gauss-Volterra principle of 'natural selection' in competitive system. We thus see that the process of natural selection lies in the increase of complexity. We have proved the increase of complexity given by (3.5) with the increase of number of species and therefore with the number of differential equations describing the dynamics of the system. The validity of the above statement requires two conditions to be satisfied. First, the system must be at stable stationary states just before the appearance of any new species and secondly, the system must be unstable just after the appearance of the new species. However, if any one of the conditions fails, the increase of complexity with the increase of the number of differential equations does not materialize. We shall illustrate this with a model system. Let us consider Rossler model system which is the simplest possible strange attractor. The system is described by the system of three differential equations [16]

$$
\begin{aligned}
& \dot{x}=-y-z \\
& \dot{y}=x+a y \\
& \dot{z}=b+z(x-c)
\end{aligned}
$$

For the choice of parameters $a=0.1 ; b=0.1$ and $c=$ 14 there is apparent chaotic attractor. The Lyapunov exponents have been determined by computation simulation to be approximately $0.072,0$ and -13.79 . The first two equations of the system are linear. We begin by looking at the dynamics in the $x y$-plane only. Setting $z=$ 0 yields 


$$
\begin{aligned}
& \dot{x}=-y \\
& \dot{y}=x+a y
\end{aligned}
$$

The origin $(0,0)$ is a stationary point. The eigenvalues of the Jacobian matrix at $(0,0)$ are $\left[a \pm \sqrt{a^{2}-4}\right]^{\frac{1}{2}}$. For $a>0$, there is at least one eigenvalue with positive real part (or positive Lyapunov exponents). So the origin is unstable. We may consider the system (4.8) to represent the dynamics of a system of two species $\mathrm{x}$ and $\mathrm{y}$ having unstable stationary state $(0,0)$. The system $(4.7)$ may be considered as the enlarged form of the system (4.8) when a new species $\mathrm{z}$ is introduced in the original system (4.8). The measure of complexity of the system (4.7) according to the formula (3.5) is given by $C_{2}=0.072$ and that of the system (4.8) is $C_{1}=0.1$. We thus see the decrease of complexity in spite of the increasing of the number of differential equations. This is in view of instability of the stationary state of the original system (4.8). As such Rossler system (4.7) can not serve as a mathematical model for biological evolution.

\section{Conclusions}

The main objectives of the paper is to study the interrelationship between the concepts of dynamical stability and complexity and to study the importance of this relationship in an evolving biological system on the basis of dynamical model (both deterministic and chaotic) of the system described by a set of kinetic equations. The characteristic features and results of the paper are as follows:

1) We have started with the dynamical model of a biological system consisting of a number of interacting biopolymer species (or ecological species). The study of dynamical stability and dynamical complexity is confined to the local behaviour or analysis of the system around a stationary or reference state.

2) A significant step in the characterization of the statistical and chaotic behaviours of the evolving system near a stationary or reference state lies in the use of a Boltzmann-like entropy (3.4) which is valid subject to the local character of the system around a stationary state or fixed point with a certain value of the control parameter $\alpha$ [1,23]. A completely different approach to the Boltzmannlike entropy (3.4) is provided by the entropy of the evolution matrix [14].

3) Another significant result is the expression of Boltzmann-entropy production rate leading to the well-known Pesin-type identity which provides an elegant and simple measure of the dynamical complexity (3.5) in terms of positive Lyapunov exponents.

4) The dependence of the measure of complexity (3.5) on the positive Lyapunov exponents (or positivity of real parts of eigenvalues) makes it very easy to understand the relationship between the concepts of dynamical stability and dynamical complexity. This stability-complexity relationship is of significant importance in the study of evolution of the system.

5) The expression of dynamical complexity (3.5) which is the sum of positive Lyapunov exponents is very helpful in the study of the increase of complexity with the instability resulting from the appearance of a new species into the system at stable steady state. This is an advantageous point with the expression of dynamical complexity (3.5).

6) The increase of the number of species or increase of the number of differential equations describing the system does not always imply the increase of the dynamical complexity. The increase of dynamical complexity is subject to two conditions to be satisfied. Violation of any one of these conditions results in the failure of the increase of complexity. In Section 4 we have explained it with an illustrative example.

7) In Section 4, using a simple competitive model system we have illustrated the increase of dynamical complexity with the successive instabilities. We have also shown how the increase of dynamical complexity leads to Gauss-Volterra "principle of natural selection" for the survival of the fittest [6]. The present study of biological evolution on the basis of concepts of stability, entropy and complexity is in the spirit of the principle of "order through fluctuation" [1-3].

\section{Acknowledgements}

The authors wish to thank the learned referees for their valuable comments and suggestions for the modification and revision of the paper. The work was done under a major research project sanctioned by U.G.C (India).

\section{References}

[1] G. Nicolis and I. Prigogine, "Self-Organization in Non-EQuilibrium Systems," Wiley and Sons, New York, 1977.

[2] I. Prigogine and G. Nicolis, "Biological Order, Structure and Instability," Quarterly Review of Biophysics, Vol. 4, No. 2-3, 1971, pp. 107-148.

[3] I. Prigogine, G. Nicolis and A. Babloyantz, "Thermodynamics of Evolution. Part-1," Physics Today, Vol. 25, No. 11, 1972, p. 23; "Thermodynamics of Evolution. Part-2," Physics Today, Vol. 25, No. 11, 1972, p. 38. doi:10.1063/1.3071140

[4] L. Demetrius, "Thermodynamics and Evolution," Journal of Theoretical Biology, Vol. 206, No. 1, 2000, pp. 1-16. doi:10.1006/jtbi.2000.2106

[5] B. H. Weber, D. J. Depew and J. D. Smith, "Entropy, Information and Evolution," MIT Press, Cambridge, 
2000.

[6] R. Feistel and W. Ebeling, "Evolution of Complex System," Kluwer Academic Publisher, Dordrecht, 1989.

[7] W. Ebeling and R. Feistel, "Theory of Self-Organization and Evolution. The Role of Entropy, Value and Information," Journal of Non-Equilibrium Thermodynamics, Vol. 17, No. 4, 1992, pp. 303-332.

[8] T. S. Ray, "Evolution, Complexity and Artificial Reality," Physica D, Vol. 75, No. 1-3, 1994, pp. 239-263. doi:10.1016/0167-2789(94)90286-0

[9] B. Drossel, "Biological Evolution and Statistical Physics," Advances in Physics, Vol. 50, No. 2, 2001, pp. 209-295. doi:10.1080/00018730110041365

[10] N. H. Barton and J. Coe, "In the Application of Statistical Physics to Evolutionary Biology," Journal of Theoretical Biology, Vol. 259, No. 2, 2009, pp. 317-324. doi:10.1016/j.jtbi.2009.03.019

[11] C. G. Chakrabarti and S. Ghosh, "Statistical Mechanics of Complex System," Indian Journal of Theoretical Physics, Vol. 48, 1995, p. 43; "Entropic Models and Analysis of Complex Systems," System Analysis, Model and Simulation, Vol. 23, 1996, p. 103.

[12] C. G. Chakrabarti and Koyel Ghosh, "Maximum-Entropy Principle: Ecological Organization and Evolution," Journal of Biological Physics, Vol. 36, No. 2, 2009, pp. 175-183.

[13] S. Loyld and H. Pagels: "Complexity and Thermodynamic Depth," Annals of Physics, Vol. 188, No. 1, 1988, pp. 186-213. doi:10.1016/0003-4916(88)90094-2

[14] G. Jumarie, "Maximum Entropy, Information without Probability and Complex Fractals," Kluwer Academic Publishers, Dordrecht, 2000.

[15] S. H. Strogatz, "Non-Linear Dynamics and Chaos," Ad-
dison-Weslay Publishing Co. Inc, New York, 1994.

[16] K. T. Aligood, T. D. Sauer and J. A. Yorke, "Chaos: An Introduction to Dynamical System," Springer, New York, 1997.

[17] R. C. Hilborn, "Chaos and Non-Linear Dynamics," Oxford University Press, Oxford, 2000.

[18] J. R. Dorfman, "An Introduction to Chaos and Non-Equilibrium Statistical Mechanics," Cambridge University Press, Cambridge, 1999.

[19] N. Korabel and Eli Barkai, "Pesin Type Identity for Intermittent Dynamics with Zero Lyapunov Exponent," Physical Review Letters, Vol. 102, No. 5, 2009, Article ID 050601. doi:10.1103/PhysRevLett.102.050601

[20] V. S. Anishchenko et al., "Non-Linear Dynamics of Chaotic and Stochastic Systems," Springer, New York, 2001.

[21] V. Latora and M. Baranger, "Kolmogorov-Sinai Entropy Rate Vs Physical Entropy," Physical Review Letters, Vol. 82 , No. 3, 1999, pp. 520-523. doi:10.1103/PhysRevLett.82.520

[22] C. Beck and F. Sc ḥ $1 \ddot{0}$ gl, "Thermodynamics of Chaotic Systems," Cambridge University Press, Cambridge, 1997.

[23] H. Haken, "Advanced Synergetics," Springer, New York, 1983.

[24] C. G. Chakrabarti, S. Ghosh and S. Bhadra, "Non-Equilibrium Thermodynamics of Lotka-Volterrra Ecosystem: Stability And Evolution," Journal of Biological Physics, Vol. 21, No. 4, 1995, pp. 273-284. doi:10.1007/BF00700629

[25] R. V. Sole and J. Bascompte, "Self-Organization in Complex Ecosystems," Princeton University Press, Princeton, 2004. 